\title{
Disseminated Phaeohyphomycotic Lymphadenitis with Cladophialophora Species
}

\author{
Authors: \\ Safia Moin,, S. Faisal Mahmood, ${ }^{2}$ "Joveria Farooqi,, Faheem Naqvi," \\ Romana Idress, ${ }^{3}$ Kauser Jabeen, ${ }^{1}$ Afia Zafar ${ }^{1}$ \\ 1. Department of Pathology and Laboratory Medicine, Clinical Microbiology, Aga \\ Khan University, Karachi, Pakistan \\ 2. Department of Medicine, Infectious Diseases, Aga Khan University, Karachi, \\ Pakistan \\ 3. Department of Pathology and Laboratory Medicine, Histopathology, Aga Khan \\ University, Karachi, Pakistan \\ *Correspondence to joveria.farooqi@aku.edu
}

Disclosure: The authors have declared no conflicts of interest.

Acknowledgements: The authors would like to thank Faisal Malik for photography of microscopic images of culture.

Received: $\quad 29.11 .20$

Accepted: $\quad 22.01 .21$

Keywords: Cladophialophora, phaeohyphomycosis.

Citation: $\quad$ EMJ Microbiol Infect Dis. 2021; DOI/10.33590/emjmicrobiolinfectdis/20-00276

\section{Abstract}

Objective: This study describes a case of disseminated phaeohyphomycotic lymphadenitis in a young female with delayed diagnosis and good clinical response after appropriate treatment.

Methods: A 32-year-old female presented with erythematous to violaceous papules with oozing discharge bilaterally in her inguinal region for a few months. History revealed tuberculous meningitis 4 years earlier treated with first-line anti-tuberculous therapy for 18 months, and 2 years previously she developed pigmented discharging lymph nodes bilaterally in her axillae. The histopathology of the biopsy of the axillary nodes showed chronic granulomatous inflammation with multiple branching septate fungal hyphae. She received amphotericin B for 21 days but without improvement. Biopsy from the inguinal lesions was sent for histopathology and culture.

Results: Histopathology of the biopsy material showed chronic granulomatous inflammatory process with multinucleate giant cells, epithelioid cells, histiocytes, and lymphocytes with multiple branching septate fungal hyphae. Gram stain revealed moderate septate hyphae with numerous pus cells. Culture on Sabouraud dextrose agar yielded velvety olive-black colonies in the fourth week. Microscopic slide examination of culture material was suggestive of Cladophialophora species. The patient was started on voriconazole, which was continued for 6 months, and showed clinical improvement.

Conclusion: Incomplete investigation of infectious lesions may delay diagnosis. Furthermore, clinical presentations are greatly influenced by the immune status of the host. Both histopathological and microbiological assessments are equally important for making a conclusive diagnosis. Anti-fungal therapy may delay the growth of fungi that normally grow within a week; thus, a longer incubation time may be warranted for fungal smear-positive samples. 


\section{INTRODUCTION}

Phaeohyphomycosis is an infection of the skin, subcutaneous tissues, and internal organs caused by dematiaceous (melanised) fungi found in soil, organic material, plants, and air. Histopathology of infected tissues usually reveals dematiaceous septate, regular or distorted, toruloid hyphae beside yeast-like cells. The presence of melanin in their cell walls is considered a virulence factor for these fungi. During the past several decades, phaeohyphomycosis has been attributed to over 100 species and 60 genera of fungi. Most clinically relevant species are within the genera of Scedosporium, Exophiala, Cladophialophora, Exserohilum, Bipolaris, Curvularia, Wangiella, and Lecythophora. ${ }^{1} \quad$ Although cerebral infection is the most serious form of systemic phaeohyphomycosis, other localised deep forms of the disease have been reported.

An infection is likely introduced by a traumatic inoculation, which may even be unrecognised by the patient, or inhalation of the aetiologic agent. ${ }^{2}$ Skin and subcutaneous tissue involvement are termed subcutaneous phaeohyphomycosis, which is characterised by papulonodules, verrucous, hyperkeratotic or ulcerated plaques, cysts, abscesses, pyogranuloma, and nonhealing ulcers, or sinuses. Clinical presentations are also influenced by the immune status of the host. The primary risk factor is decreased host immunity, although cases in apparently immunocompetent patients have been reported. 2,3 Phaeohyphomycoses are frequently associated with debilitating chronic diseases like diabetes, rheumatoid arthritis with prolonged corticosteroid therapy, and solid organ, especially kidney, transplant. ${ }^{4}$ Moreover, longterm immunosuppression from corticosteroids may lead to delayed clinical expression. ${ }^{5}$ While immunocompromised patients are at risk of locally invasive phaeohyphomycosis, rarely fatal complications such as pneumonia or disseminated disease may occur. Disseminated phaeohyphomycosis is an uncommon infection, although its incidence may be increasing in the population. ${ }^{1}$ Early diagnosis and careful interpretation of culture results are therefore important for the treatment of these patients. This communication reports a case of subcutaneous phaeohyphomycosis with disseminated lymphadenitis caused by Cladophialophora species.
CASE

A 32-year-old female, with a history of treated tuberculous meningitis in 2014, presented with erythematous to violaceous papules, with oozing discharge bilaterally in her inguinal region for a few months. She reported that in March 2016 she had developed similar pigmented discharging lymph nodes bilaterally in her axillae and slight enlargement of her left inguinal lymph node with minimal discharge. The axillary lymph nodes biopsy at the time showed ulcerated skin with underlying adipose tissue exhibiting dense acute and chronic inflammatory infiltrate. Focal abscess formation and necrosis with multiple branching septate fungal hyphae were also highlighted by periodic acid-Schiff and diastase special stain. The patient did not recall any fever or systemic symptoms and her erythrocyte sedimentation rate was $5 \mathrm{~mm} /$ hour at that time. She was nonreactive for HIV. She received amphotericin B for 21 days for the axillary lesions, with no response. However, in March 2018 she developed new lymph nodes. Biopsy showed non-specific inflammation. Another trial of amphotericin for 21 days was given, which failed again. Subsequently, another biopsy was taken in October 2018, this time from inguinal lesions (Figure 1A), and it was sent for histopathology and fungal culture. Histopathology revealed chronic granulomatous inflammation with multinucleated giant cells, epithelioid cells, histiocytes, and lymphocytes with multiple branching septate fungal hyphae, suggesting a deep-seated fungal infection (Figure 1B). Special stain periodic acid-Schiff with or without diastase also revealed fungal spores and hyphae. Acid-fast bacilli and WadeFite stain (for Mycobacterium leprae) were both negative.

Gram stain showed moderate pigmented septate hyphae with numerous pus cells. In the fourth week of incubation at $37^{\circ} \mathrm{C}$, culture on Sabouraud dextrose agar yielded velvety oliveblack colonies with a black reverse (Figure 1C). Microscopic slide examination of the culture on Sabouraud dextrose agar revealed dark, septate, freely branching hyphae giving rise to medium-length chains of dark staining conidia. The conidia were elliptical in shape, smooth with a prominent hilar scar, and 10-15 conidia-long chains arose from 'shield cells' where branching occurred (Figure 1D). 
The isolate failed to grow at $42{ }^{\circ} \mathrm{C}$ while it grew well at $37{ }^{\circ} \mathrm{C}$ and $25^{\circ} \mathrm{C}$ and was thus identified phenotypically as Cladophialophora species. ${ }^{6}$

The patient was initiated on voriconazole the same month, to which she gradually responded, with resolution of the axillary lesion and significant reduction in the size of the inguinal lymph node, as monitored by ultrasound. Her HIV status remained negative. Unfortunately, the patient was lost to follow-up until April 2019. During the intervening months, she had stopped the voriconazole in January 2019, leading to another relapse, and had subsequently restarted this for a month only to stop again. As she was asymptomatic, she was observed off therapy.

She developed discharge from the lesions and therapy was reinitiated in April 2019 with adherence counselling. She responded well to therapy, with a gradual reduction in the size of the nodes (as documented on ultrasound; Table 1). Voriconazole was continued up to June 2020 until stability in the lymph node size was achieved. A follow-up ultrasound is awaited. Given the severe financial constraints of the patient, a work-up for immunodeficiency could not be performed.

\section{DISCUSSION}

The authors describe a case of Cladophialophora species lymph node and cutaneous infection in an apparently immunocompetent host. Most clinically relevant species are within the genera of Exophiala, Cladophialophora, Coniosporum, Cyphellophora, Fonsecaea, Phialophora, and Rhinocladiella. Several cases of phaeohyphomycosis and chromoblastomycosis have been reported from India and Brazil. ${ }^{7-9}$

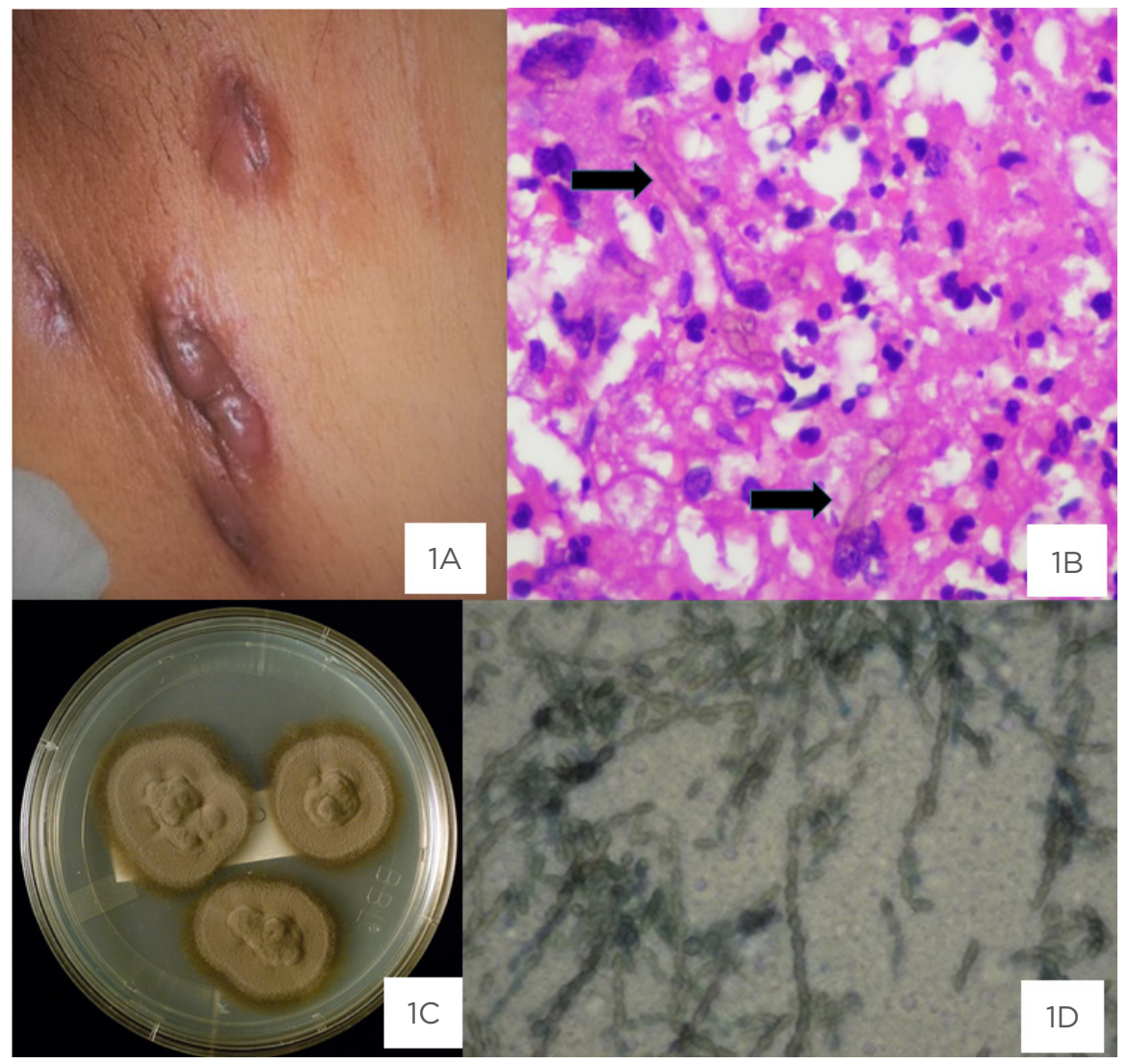

Figure 1: Image plate of clinical lesion, histopathology, macroscopic colony morphology, and microscopic appearance of the organism, Cladophialophora species. Draining sinuses in the groin and lower abdominal wall.

1A: Draining sinuses in the groin and lower abdominal wall with dark pigmented discolouration of skin. 1B: Histopathology of inguinal lymph node showing chronic granulomatous inflammation and golden brown pigmented septate hyphae with some dilated forms (arrows). 1C: Culture on Sabouraud's dextrose agar: velvety olive-black colonies at 4 weeks of incubation at 37 oC. 1D: Chains of 10-15 elliptical dark conidia with branching. 
Table 1: Progression of the lymph node size on therapy with voriconazole (initiated April 2019).

\begin{tabular}{|l|l|l|}
\hline Year & Left inguinal $(\mathrm{cm})$ & Right inguinal $(\mathrm{cm})$ \\
\hline June 2019 & $2.7 \times 1.1$ & $2.6 \times 1.0$ \\
\hline August 2019 & $2.4 \times 1.1$ & $2.1 \times 0.8$ \\
\hline November 2019 & $2.0 \times 0.8$ & $2.1 \times 0.7$ \\
\hline February 2020 & $1.8 \times 0.8$ & $1.9 \times 0.7$ \\
\hline June 2020 & $1.4 \times 0.7$ & $1.9 \times 0.7$ \\
\hline
\end{tabular}

Limited data are available from Pakistan; however, laboratory-based surveillance from the authors' institute reported cerebral phaeohyphomycosis to be as common in brain abscesses as that due to Aspergillus species. ${ }^{10}$ Moreover, melanised fungi resulted in around 9\% of all invasive skin and soft tissue infections in the data..$^{10}$ There is a scarcity of reported data from Pakistan; however, few studies report these infections mainly in immunocompetent patients. ${ }^{11,12}$ One reason for the relatively higher frequency of these moulds in low-to-middle-income agricultural countries may be the humid, tropical, and subtropical climate, which potentially facilitates the growth of these organisms in the environment..$^{13}$

The organisms are likely introduced by a minor traumatic inoculation, which is often not recognised by the patient. ${ }^{3}$ Clinical presentations are greatly influenced by the immune status of the host. Regarding the patient in this current study, a history of tuberculosis may also suggest a poor cell-mediated immune response; therefore, a trivial, unnoticed trauma may have been responsible for initiation of the infection. Additionally, the authors did not evaluate for genetic mutations such as CARD9 deficiency, which has been reported to predispose to serious fungal infections in otherwise healthy patients from the region. ${ }^{14}$

Several similar cases have been reported from Brazil. In a series of 18 cases of phaeohyphomycosis, $61 \%$ presented with subcutaneous lesions. In all of the cases, the presence of melanin in the fungal cells was determined by Fontana-Masson staining of tissue sections and documented. The causative organisms included Exophiala jeanselmei,
Alternaria, Curvularia, Cladophialophora, and Colletotrichum gloeosporioides. ${ }^{8}$

Treatment of Cladophialophora species is often difficult, prolonged, and not standardised. Itraconazole and voriconazole have a broad range of activity against phylogenetically similar agents of hyalohyphomycosis, phaeohyphomycosis, chromoblastomycosis, and mycetoma. In vitro comparison showed that the minimum inhibitory concentration (MIC) ranges (geometric mean) of amphotericin B, itraconazole, and voriconazole against Cladophialophora carrionii were 0.06 $4.00 \mu \mathrm{g} / \mathrm{mL}$ (1.07 $\mu \mathrm{g} / \mathrm{mL}), 0.03-0.06 \mu \mathrm{g} / \mathrm{mL}$ $(0.03 \mu \mathrm{g} / \mathrm{mL})$, and $0.030-0.125 \mu \mathrm{g} / \mathrm{ml}(0.05 \mu \mathrm{g} /$ $\mathrm{mL}$ ), respectively. ${ }^{15}$ Another collection of $81 \mathrm{C}$. carrionii strains revealed that itraconazole and posaconazole had the lowest MIC required to inhibit $90 \%$ of the strains tested $\left(\mathrm{MIC}_{90}: 0.063\right.$ $\mu \mathrm{g} / \mathrm{mL})$, followed by terbinafine ( $\mathrm{MIC}_{90}: 0.125 \mu \mathrm{g} /$ $\mathrm{mL}$ ) and isavuconazole and voriconazole ( $\mathrm{MIC}_{90}$ : $0.25 \mu \mathrm{g} / \mathrm{mL}$ ). The highest values were recorded for caspofungin $\left(\mathrm{MIC}_{90}: 2 \mathrm{\mu g} / \mathrm{mL}\right.$ ), micafungin $\left(\mathrm{MIC}_{90}: 4 \mu \mathrm{g} / \mathrm{mL}\right)$, amphotericin $\mathrm{B}\left(\mathrm{MIC}_{90}: 8 \mu \mathrm{g} /\right.$ $\mathrm{mL})$, and fluconazole $\left(\mathrm{MIC}_{90}: 64 \mu \mathrm{g} / \mathrm{mL}\right){ }^{16} \mathrm{~A}$ systematic review by Hellwig et al. ${ }^{17}$ examined 35 studies evaluating the susceptibility of chromoblastomycosis agents to eight antifungal drugs and their combinations. Posaconazole, terbinafine, itraconazole, and voriconazole were, in descending order, the most effective antifungal drugs against chromoblastomycosis agents in vitro. In combination, only terbinafine plus voriconazole and itraconazole plus caspofungin showed $100 \%$ synergy for Fonsecaea pedrosoi, Exophiala jeanselmei, and Phialophora verrucosa, while none showed antagonism. 
Given the paucity of clinical data, treatment of phaeohyphomycosis depends on the clinical syndrome and the immune status of the patient. For localised subcutaneous nodules, surgery alone may be curative. Itraconazole has excellent activity and has been used most, although voriconazole and posaconazole are increasingly being used with good results. ${ }^{18}$ Amphotericin $B$ is often ineffective. The duration of therapy varies but may range from 6 weeks to more than 12 months. For this patient, since the disease was disseminated and the possibility of an immune deficiency could not be ruled out, treatment with voriconazole and close monitoring of the number and size of lesions through ultrasound was continued for 21 months.

\section{CONCLUSION}

Despite a difficult diagnosis and a rare occurrence, physicians and surgeons should be aware of this infection, especially in immunocompromised patients. It can often be misdiagnosed as a carcinomatous, non-healing ulcer. Incomplete investigation of infectious lesions may delay diagnosis. Both histopathological and microbiological assessments are equally important for making a conclusive diagnosis. As antifungal therapy may delay growth of fungi that normally grow within a week, a longer incubation time may be warranted for suspected samples.

Surgery in combination with prolonged treatment with triazoles is effective for the eradication of phaeohyphomycosis.

\section{References}

1. Revankar SG et al. Disseminated phaeohyphomycosis: review of an emerging mycosis. Clin Infect Dis. 2002;34(4):467-76.

2. Chen YC et al. Subcutaneous phaeohyphomycosis caused by Exophiala jeanselmei. J Microbiol, Immunol Infect. 2014;47(6):546-9.

3. Badali $\mathrm{H}$ et al. Subcutaneous phaeohyphomycotic cyst caused by Pyrenochaeta romeroi. Med Mycol. 2010;48(5):763-8.

4. Haridasan S et al. Subcutaneous phaeohyphomycosis in kidney transplant recipients: a series of seven cases. Transpl Infect Dis. 2017;19(6):e12788.

5. Sharma $S$ et al. Subcutaneous phaeohyphomycosis caused by Pyrenochaeta romeroi in a rheumatoid arthritis patient: a case report with review of the literature. Mycopathologia. 2016;181(9-10):73543.

6. Larone DH. Medically Important Fungi: A Guide to Identification (2002) 4th edition, Washington DC: American Society for Microbiology Press.

7. Shirbur SN et al. Recurrent phaeohyphomycosis: a case report. J Clin Diagn Res. 2013;7(9):2015-6.

8. Severo CB et al. Phaeohyphomycosis: a clinical-epidemiological and diagnostic study of eighteen cases in Rio Grande do Sul, Brazil. Mem Inst Oswaldo Cruz. 2012;107(7):854-8.

9. Mouchalouat MDF et al. Case Report. Cladophialophora carrionii: a rare agent of chromoblastomycosis in Rio de Janeiro state, Brazil. Rev Inst Med trop S Paulo. 2008;50(6):351-3.

10. Jabeen $\mathrm{K}$ et al. Spectrum of invasive fungal infections from Pakistan; laboratory based surveillance data (2009-2014). Abstract P190. 8th Trends in Medical Mycology, EORTC and ECMM, 6-9 October, 2017.

11. Ajanee $\mathrm{N}$ et al. Brain abscess caused by Wangiella dermatitidis: case report. Clin Infect Dis. 1996;23(1):1978.

12. Jabeen $\mathrm{K}$ et al. Rhinocladiella mackenziei as an emerging cause of cerebral phaeohyphomycosis in Pakistan: a case series. Clin Infect Dis. 2011;52(2):213-7.

13. Deng $S$ et al. Global spread of human chromoblastomycosis is driven by Recombinant Cladophialophora carrionii and predominantly clonal Fonsecaea species. PLoS Negl Trop Dis. 2015;9(10):e0004004.

14. Vaezi A et al. Frequency and geographic distribution of CARD9 mutations in patients with severe fungal infections. Front Microbiol. 2018;9:2434.

15. McGinnis MR, Pasarell L. In vitro testing of susceptibilities of filamentous ascomycetes to voriconazole, itraconazole, and amphotericin B, with consideration of phylogenetic implications. J Clin Microbiol. 1998;36(8):2353-5.

16. Deng $\mathrm{S}$ et al. In vitro antifungal susceptibility of Cladophialophora carrionii, an agent of human chromoblastomycosis. Antimicrob Agents Chemother. 2013;57(4):1974-7.

17. da Silva Hellwig $\mathrm{AH}$ et al. In vitro susceptibility of chromoblastomycosis agents to antifungal drugs: a systematic review. J Glob Antimicrob Resist. 2019;16:10814.

18. Revankar SG et al. A mycoses study group international prospective study of phaeohyphomycosis: an analysis of 99 proven/probable cases. Open Forum Infect Dis. 2017;4(4):ofx200. 